Journal of Social Sciences 7 (3): 375-379, 2011

ISSN 1549-3652

(C) 2011 Science Publications

\title{
Are Improvements in Physical Fitness after an Exercise Program Related to the Symptomatology in Patients with Fibromyalgia
}

\author{
${ }^{1}$ Borja Sanudo, ${ }^{1}$ Luis Carrasco, ${ }^{1}$ Moisés de Hoyo and ${ }^{2}$ Nicolae Ochiana \\ ${ }^{1}$ Department of Physical Education and Sport, University of Seville, Spain \\ ${ }^{2}$ Department of Sports and Human Movement Sciences, Faculty of Health, \\ University "Vasile Alecsandri" of Bacau, Romania
}

\begin{abstract}
Problem statement: Physical exercise is considered one of the main strategies in management of Fibromyalgia (FM); While many studies have shown relevant improvements in the patients' physical condition and psychological status, the efficacy of these treatments have not yet been completely determined and whether or not these improvements are related with symptom severity is still debated. The aim of this study was to evaluate the positive effect of exercise training on physical fitness and second to determine whether any change in aerobic capacity, strengthening or flexibility after exercise training is associated with improvements in symptoms severity of patients with FM. Approach: Thirty women with FM were randomized to either combined exercise group $(\mathrm{CE}, \mathrm{n}=15)$ which performed twice-weekly sessions of combined aerobic and resistance exercises, or usual care control group (CG, $\mathrm{n}=15$ ). Participants had to complete a Visual Analogue Scale (VAS) for stiffness, sleep disturbances, pain, anxiety and depression. Physical fitness (aerobic capacity, handgrip strength and flexibility) was also assessed at baseline and immediately after 12-week intervention. Results: Aerobic capacity and Range Of Motion (ROM) in both shoulders and in the right hip were significantly greater than controls. Inter-group differences were significant in anxiety and depression. Linear regressions of physical fitness variables and symptomatology showed positive correlations. Conclusions: Physical fitness seems to be related with the improvements in symptom' severity and due to regular physical activity has demonstrated to be beneficial for individuals with FM it should be included in treatment plans for this patients group.
\end{abstract}

Key words: Physical exercise, Visual Analogue Scale (VAS), symptomatology, physical fitness, fatigue, symptoms severity, flexibility exercises, individuals, scientific evidence, Range of Motion (ROM), Analysis of Variance (ANOVA)

\section{INTRODUCTION}

Fibromyalgia is a chronic rheumatic condition consisting of diffuse musculoskeletal pain, fatigue, sleep disturbances and tenderness on palpation (Wolfe et al., 1990). While the concept of central sensitization is increasingly accepted as an important pathophysiologic aberration in FM patients, the factors that contribute to its persistence are not wellcharacterized (Price and Staud, 2005). FM patients have low levels of physical fitness and often experience postexertional pain, fatigue and delayed onset of muscle soreness (Jones et al., 2006).

Conventional medical treatment generally produces modest and inconsistent benefits on symptoms, functioning and quality of life (Gore et al., 2009); therefore, nonpharmacologic treatments, such as exercise and cognitive-behavioral interventions, are recommended to assist people with FM to better manage the array of symptoms and functional deficits (Gore et al., 2009). Scientific evidence supports the use of physical exercise program for the overall management of this population group (Busch et al., 2008). Improvements have been reported among others in pain relief, sleep quality, stiffness, anxiety or depression (Assis et al., 2006; Bircan et al., 2008; Gusi et al., 2006; Tomas-Carus et al., 2005 Valkeinen et al., 2008) . Despite this, the symptoms of FM often create obstacles that deter many from exercising consistently enough to derive benefits (Jones and Liptan, 2009). Thus, finding new ways to promote increased physical activity in persons with FM that can be sustained overtime is important. Therefore the aim of the current study was twofold, first to assess whether the exercise training have any effect on physical fitness and second 


\section{J. Social Sci., 7 (3): 375-379, 2011}

to determine whether any change in aerobic capacity, strengthening or flexibility after exercise training is associated with improvements in symptoms severity.

\section{METERIALS AND METHODS}

Participants: A total of 30 women who met the American College of Rheumatology criteria for classification of FM (Wolfe et al., 1990) had to give written informed consent to be involved in the study. Participants were randomized to either combined exercise group (CE, $\mathrm{n}=15)$ or usual care control group $(\mathrm{CG}, \mathrm{n}=15)$.

The exclusion criteria included the presence of inflammatory rheumatic diseases and severe psychiatric illness. Participants with respiratory or cardiovascular diseases that prevent physical exertion were also omitted. Finally, women with FM receiving psychological or physical therapy were excluded to avoid possible interactions with the present trial. This research was approved by the University of Seville Research Ethics Committee.

Outcome measures: Assessment of outcomes was undertaken at baseline and immediately after the 12week intervention and at the same time points in the usual care control group. The primary outcomes of the study were the change in physical fitness and symptoms severity.

Symptoms: Subjects completed a Visual Analogue Scale (VAS) for stiffness, sleep disturbances, pain, anxiety and depression. Each VAS is a $10 \mathrm{~cm}$ scale where 0 represents an absence of the symptom and 10 represents the possible maximum value for the symptom.

Physical fitness: The (6MWT) was used to estimate aerobic capacity and has been shown to be a reliable and valid measure in fibromyalgia patients. Hand-grip strength was assessed in both hands with a handdynamometer (TKK, Tokyo, Japan) and range of motion (ROM- flexion/extension) in the shoulders and hips were assessed using a manual goniometer (Lafayette Instrument Co., Lafayette, Indiana), with the best of three trials being recorded.

Procedures: Participants randomized to CE performed twice-weekly sessions of combined aerobic and resistance exercises, including a 10 min warm up, 10-15 min of aerobic exercise at $65-70 \%$ HRmax, 15-20 min of muscle strengthening exercises (one set of 8-10 repetitions for eight different muscle groups, with a 1-3 $\mathrm{kg}$ load) and $10 \mathrm{~min}$ of flexibility exercises (one set of three repetitions for 8-9 different exercises, maintaining the stretched position for $30 \mathrm{~s}$ ). The $\mathrm{CG}$ was receiving medical treatment for FM and continued their normal daily activities during the period of the intervention.

Statistical analyses: The results are expressed as the mean \pm SD. Changes in the dependent variables over time within groups and differences between groups at the same time points were evaluated using repeatedmeasures Analysis Of Variance (ANOVA). To assess the possible relation between variables, linear regression analysis between physical fitness and symptomatology were performed. The significance level was determined at $\mathrm{p}<0.05$. Statistical analysis was carried out using SPSS for Windows 15 (SPSS, Chicago, IL).

\section{RESULTS}

One woman from the exercise group and another one from the usual care control group were lost to follow-up. Reasons for drop out included illness and one of the patients did not attend the final evaluation session. Table 1 shows the baseline characteristics of the two groups. The groups were well-matched at the baseline assessment, with no differences in any of the key outcome variables being apparent.

Physical fitness: In CE, joint mobility in both shoulders $(\mathrm{P} \leq 0.013)$ and in the right hip $(\mathrm{P}<0.014)$ were significantly greater than controls after 12-weeks intervention, improvement in 6MWT distance were also observed in this patient group $(\mathrm{p}=0.044)$. However inter-groups differences were not significant for handgrip strength.

Symptoms: Improvements were significant for sleep disturbances $(p=0.04)$ and also for pain $(p=0.03)$ and there was a trend toward a better stiffness in CE $(23 \%$, $\mathrm{p}=0.06)$. Lower benefits were found for anxiety $(22 \%)$ or depression $(21 \%)$. No differences were found in CG. Regarding the inter-group differences, there were just significant differences between $\mathrm{CE}$ and $\mathrm{CG}$ in anxiety and depression.

Physical fitness-Symptoms association: Linear regressions of physical fitness variables and symptomatology are shown in Fig. 1, with positive correlations noted between sleep disturbances and the ROM in both right $(r=0.49)$ and left arm $(r=0.44)$ and between anxiety and the ROM in the right arm ( $\mathrm{r}=$ 0.40). Improvements in strength were also correlated with depression $(\mathrm{r}=0.43-0.51)$ with both hands and with pain $(r=0.60)$ after the 12 weeks intervention. 
J. Social Sci., 7 (3): 375-379, 2011

Table 1: Effects of a 12-weeks exercise program on physical fitness and symptoms severity

\begin{tabular}{|c|c|c|c|c|c|c|}
\hline & \multirow[b]{2}{*}{ Outcomes } & \multicolumn{3}{|c|}{ Baseline mean (SD) } & \multicolumn{2}{|l|}{ Postest at 12 weeks } \\
\hline & & GE & $\mathrm{GC}$ & $\mathrm{P}$ & Mean $(95 \% \mathrm{CI})$ & $\begin{array}{l}\text { Inter-group } \\
\text { differences (p) }\end{array}$ \\
\hline \multirow[t]{5}{*}{ Symptoms } & Stifness & $5,93(2.81)$ & $6,21(3.02)$ & 0.798 & $1.36(-0.86,2.80)$ & 0.120 \\
\hline & Sleep disturbances & $6,93(2.76)$ & $7,71(2.64)$ & 0.449 & $0.78(0.28,1.54)$ & 0.131 \\
\hline & Pain & $7,43(1.40)$ & $6,86(1.70)$ & 0.341 & $0.78(0.63,1.51)$ & 0.369 \\
\hline & Anxiety & $6,14(3.23)$ & $7,43(2.62)$ & 0.258 & $1.36(-1.10,3.81)$ & $0.019^{*}$ \\
\hline & Depression & $5,21(3.12)$ & $7,14(2.71)$ & 0.093 & $1.07(-0.26,2.40)$ & $0.014 *$ \\
\hline \multirow[t]{7}{*}{ Physical fitness } & Right leg ROM & $73,36(10.89)$ & $65,5(13.51)$ & 0.102 & $-2.28(-8.93,4.36)$ & $0.014 *$ \\
\hline & Left leg ROM & $69,86(8.83)$ & $70,57(13.15)$ & 0.867 & $-4.86(-10.37,0.66)$ & 0.276 \\
\hline & Right arm ROM & $147,07(24.52)$ & $135,57(18.68)$ & 0.175 & $-9.07(-17.71,-0.43)$ & $0.013 *$ \\
\hline & Left arm ROM & $153,07(18.42)$ & $141,5(14.32)$ & 0.075 & $-6.93(-15.00,1.15)$ & $0.001 *$ \\
\hline & 6MWTD & $548,84(63.63)$ & $519,7(22.88)$ & 0.119 & $-6.14(-29.70,17.41)$ & $0.044 *$ \\
\hline & Right strength & $17,83(4.21)$ & $21,18(5.56)$ & 0.084 & $-1.30(-2.85,0.25)$ & 0.873 \\
\hline & Left strength & $14,82(5.01)$ & $18,29(6.90)$ & 0.140 & $-1.20(-3.70,1.30)$ & 0.140 \\
\hline
\end{tabular}

95\% CI: 95\% confidence interval. ROM: Range of motion; 6MWTD: Six minutes walk test distance

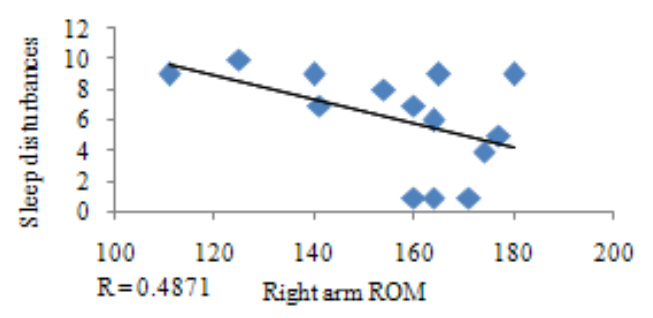

(a)

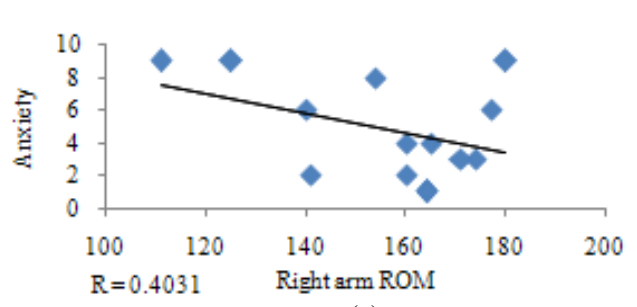

(c)

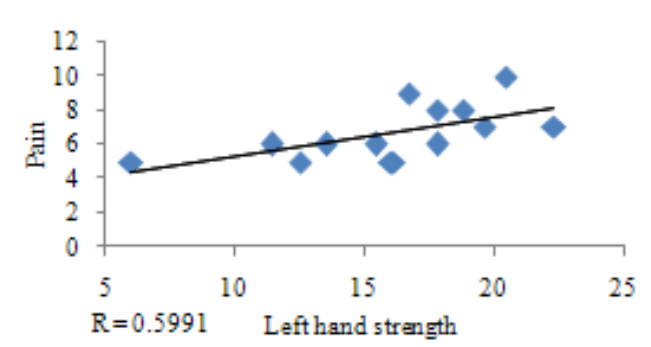

(e)



(b)

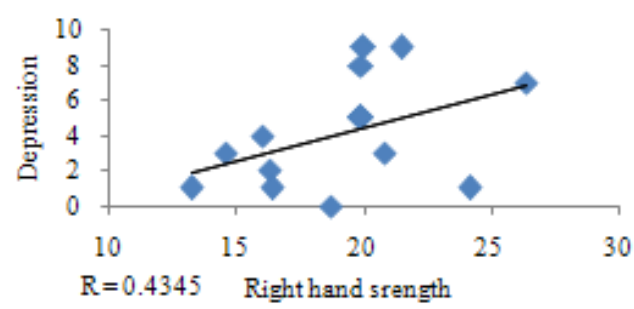

(d)



(f)

Fig. 1a-f: Linear regression analysis between physical fitness and symptomatology

\section{DISCUSSION}

The aim of this study was to evaluate the effectiveness of physical exercise on physical fitness and determine whether any change in aerobic capacity, ROM or strengthening after exercise training is associated with improvements in symptoms severity in women diagnosed with FM. This study showed that 12week exercise program led to significant gains in aerobic performance as measured by 6MWT, muscle strength as measured by hand-grip strength and flexibility as assessed by flexion-extension ROM in 


\section{J. Social Sci., 7 (3): 375-379, 2011}

hips and shoulders. In addition, the training proved to be effective in improving symptoms severity, mainly anxiety and depression.

Numerous studies have evaluated the effects of exercise programs on fibromyalgia symptoms (Häuser et al., 2009). Previous authors have compared different exercise interventions. Richards and Scott (2002), for example, compared an aerobic exercise program with another based on flexibility exercises. After 12 weeks intervention, these authors showed improvement in self reported health status in both groups, while psychological outcomes remained unchanged. In the study by Gowans et al. (2004), no significant improvements were noted in depression and anxiety subscales of FIQ in patients receiving a 6-week aerobic exercise and educational program. Longer trials that used aerobic exercise (Assis et al., 2006) or a combination of exercise and education (Valim et al., 2003) found significant improvements in psychological status. However, the improvements in anxiety and depression showed in the current study let us to think that the intensity and duration selected may be enough to induce benefits in these patients psychological status.

In the other hand, exercise training in low to moderate volume improves physical fitness in patients with FM (Valkeinen et al., 2008). It has been demonstrated that strength training can also induce cardiovascular benefits and a number of authors have reported that the combination of resistance training and aerobic exercise yields greater improvements in the cardiovascular endpoints of exercise performance compared aerobic training alone (Pierson et al., 2001). Increments in six-minute walk distance were reported after short-term exercise programs (Bircan et al., 2008) and it was determined that the large increase in the distance walked may be due in part to the increase in the participant' muscular strength. However, perhaps because of the lower frequency of strength training in our present study, the overall strength development remained relatively small. Finally, Jones et al. (2002) reported that flexibility training alone resulted in overall improvements, although there is no evidence about the real benefits of flexibility programs (Busch et al., 2008). However, the current study reached significant improvements in the ROM of shoulders and hips just with $10 \mathrm{~min} / \mathrm{session}$ of flexibility exercises.

When assessing if physical fitness is associated with improvements in symptoms severity, there is no clear consensus in the literature. Valim et al. (2003) found no correlation between improving cardiorespiratory fitness and improving quality of life, while Gowans et al. (2003) found a significant correlation between amount of physical training and depression. Gusi et al. (2006) suggested that sufficient intensity, length and frequency of training is needed to create positive effects of exercise on mood while Valim et al. (2003) suggested that training intensity would not be the determinant for this improvement. The current study found correlations between ROM and hand strength with sleep disturbances, pain, anxiety or depression what may indicate that the differences among the studies may be due to differences in duration of intervention, intensity of training and content of the programs.

\section{CONCLUSION}

In summary, due to regular physical activity has demonstrated to be beneficial in physical, psychological and functional capacity for individuals with FM and physical fitness seems to be related with the improvements in symptom' severity those programs should be included in treatment plans for these patients.

\section{REFERENCES}

Assis, M.R., L.E. Silva, A.M. Alves, A.P. Pessanha and V. Valim et al., 2006. A randomized controlled trial of deep water running: clinical effectiveness of aquatic exercise to treat fibromyalgia. Arthritis. Rheum., 55: 57-65. DOI: 10.1002/ART.21693

Bircan, C., S.A. Karasel, B. Akgün, O. El, S. Alper, 2008. Effects of muscle strengthening versus aerobic exercise program in fibromyalgia. Rheumatol. Int., 28: 527-532. DOI: 10.1007/S00296-007-0484-5 PMid:17982749

Busch, A.J., C.L. Schacter, T.J. Overend, P.M. Peloso, A. Barber, 2008. Exercise for fibromyalgia: a systematic review. J. Rheum., 35: 1130-1144. PMID: 18464301

Gore, M., A.B. Sadosky, G. Zlatera, D.J. Clauw, 2009. Clinical characteristics, pharmacotherapy and healthcare resource use among patients with fibromyalgia newly prescribed gabapentin or pregabalin. Pain. Pract., 9:363-374. DOI: 10.1111/J.1533-2500.2009.00292.X PMID: 19500273

Gowans, S.E., A. deHueck, S. Voss, A. Silaj and S.E. Abbey, 2001. Effect of a randomized, controlled trial of exercise on mood and physical function in individuals with fibromyalgia. Arthritis. Rheum., 45: 519-529. DOI: 10.1002/15290131(200112)45:6<519::AID-ART377>3.0.CO;23 PMID: 11762686

Gowans, S.E., A. Dehueck, S. Voss, A. Silaj and S.E. Abbey, 2004. Six-month and one-year followup of 23 weeks of aerobic exercise for individuals with fibromyalgia. Arthritis. Rheum., 51: 890-898. DOI: 10.1002/ART.20828 PMID: 15593364 
Gusi, N., P. Tomas-Carus, A. Häkkinen, K. Häkkinen and A. Ortega-Alonso, 2006. Exercise in waisthigh warm water decreases pain and improves health-related quality of life and strength in the lower extremities in women with fibromyalgia. Arthritis. Rheum., 55: 66-73. DOI: 10.1002/ART.21718 PMID: 16463415

Häuser, W., K. Bernardy, B. Arnold, M. Offenbächer and M. Schiltenwolf, 2009. Efficacy of multicomponent treatment in fibromyalgia syndrome: a meta-analysis of randomized controlled clinical trials. Arthritis. Rheum., 61: 216-224. DOI: 10.1002/ART.24276 PMID: 19177530

Jones, K.D, D. Adams, K. Winters-Stone and K.S. Burckhardt, 2006. A comprehensive review of 46 exercise treatment studies in fibromyalgia (19882005). Health. Qual. Life. Outcomes., 4:67-67. DOI: $10.1186 / 1477-7525-4-67$ PMID:16999856 PMCid:1590013

Jones, K.D. and G.L. Liptan, 2009. Exercise Interventions in Fibromyalgia: Clinical Applications from the Evidence. Rheum. Dis. Clin. North. Am., 35: 373-391. DOI: 10.1016/J.RDC.2009.05.004 PMID:19647149

Jones, K.D., C.S. Burckhardt, S.R. Clark, R.M. Bennett, K.M. Potempa, 2002. A randomized controlled trial of muscle strengthening versus flexibility training in fibromyalgia. J. Rheumatol., 29: 10411048. PMID: 12022321

Pierson, L.M., W.G. Herbert, H.J Norton, et al., 2001. Effects of combined aerobic and resistance training versus aerobic training alone in cardiac rehabilitation. J. Cardiopulm. Rehabil., 21: 101110. DOI: 10.1097/00008483-200103000-00007 PMID: 11314283 PMID: 16463414

Price, D.D., R. Staud, 2005. Neurobiology of fibromyalgia syndrome. J. Rheumatol. Suppl., 75:22-28. PMID: 16078357
Richards, S.C., D.L. Scott, 2002. Prescribed exercise in people with fibromyalgia: parallel group randomised controlled trial. BMJ., 325: 185-187. DOI: 10.1136/BMJ.325.7357.185 PMID:12142304 PMCID:117444

Sañudo, B., D. Galiano, L. Carrasco, M. Blagojevic and M. de Hoyo et al., 2010. Aerobic exercise versus combined exercise therapy in women with fibromyalgia syndrome: A randomized controlled trial. Arch. Phys. Med. Rehabil., 91: 1838-1843. DOI: 10.1016/J.APMR.2010.09.006 PMID: 21112423

Tomas-Carus, P., N. Gusi, A. Häkkinen, K. Häkkinen, A. Leal, A. Ortega-Alonso, 2008. Eight months of physical training in warm water improves physical and mental health in women with fibromyalgia: a randomized controlled trial. J. Rehabil. Med., 40: 248-252. DOI: $\quad 10.2340 / 16501977-0168$ PMID: 18382819

Valim, V., L. Oliveira, A. Suda, L. Silva and M. de Assis et al., 2003. Aerobic fitness effects in fibromyalgia. J. Rheumatol., 30:1060-1069. PMID: 12734907

Valkeinen, H., M. Alén, A. Häkkinen, P. Hannonen, K. Kukkonen-Harjula, K. Häkkinen, 2008. Effects of concurrent strength and endurance training on physical fitness and symptoms in postmenopausal women with fibromyalgia: a randomized controlled trial. Arch. Phys. Med. Rehabil., 89(9):1660-6. DOI: 10.1016/J.APMR.2008.01.022 PMID: 18675392

Wolfe, F., H.A. Smythe, M.B. Yunus, et al., 1990. The american college of rheumatology 1990 criteria for the classification of fibromyalgia. report of the multicenter criteria committee. Art. Rheum., 33:160-72. DOI: 10.1002/ART.1780330203 